\title{
BANACH ALGEBRAS OF VECTOR-VALUED FUNCTIONS
}

\author{
AZADEH NIKOU \\ Department of Mathematics, Tarbiat Moallem University, 599 Taleghani Avenue, Tehran 15618, Iran \\ e-mail:a_nikou@tmu.ac.ir
}

and ANTHONY G. O'FARRELL

Department of Mathematics and Statistics, NUI, Maynooth, Co. Kildare, Ireland

e-mail:admin@maths.nuim.ie

(Received 7 November 2012; revised 19 March 2013; accepted 10 May 2013; first published online 13 August 2013)

\begin{abstract}
We introduce the concept of an $E$-valued function algebra, a type of Banach algebra that consists of continuous $E$-valued functions on some compact Hausdorff space, where $E$ is a Banach algebra. We present some basic results about such algebras, having to do with the Shilov boundary and the set of peak points of some commutative $E$-valued function algebras. We give some specific examples.
\end{abstract}

2010 Mathematics Subject Classification. 46H99.

1. Introduction and preliminaries. We consider only algebras over the field of complex numbers, $\mathbb{C}$. A Banach algebra is an algebra equipped with a submultiplicative norm with respect to which it is complete. See $[4,15]$ for background on Banach algebras.

1.1. $E$-valued function algebras. Let $X$ be a non-empty compact Hausdorff space, $E$ be a unital Banach algebra and $C(X, E)$ be the space of all continuous maps from $X$ into $E$. We define the uniform norm on $C(X, E)$ by

$$
\|f\|_{X}:=\sup _{x \in X}\|f(x)\|, \quad \forall f \in C(X, E) .
$$

For $f, g \in C(X, E)$ and $\lambda \in \mathbb{C}$, the pointwise operations $\lambda f, f+g$ and $f g$ in $C(X, E)$ are defined as usual. It is easy to see that $C(X, E)$, equipped with the norm $\|\cdot\|_{X}$, is a Banach algebra. If $E=\mathbb{C}$, we get the ordinary uniform function algebra $C(X):=$ $C(X, \mathbb{C})$ of all continuous complex-valued functions on $X$. See any of $[\mathbf{2}, \mathbf{4}, \mathbf{6}, \mathbf{1 3}]$ for background on uniform algebras.

Definition 1.1. By an $E$-valued function algebra on $X$ we mean a subalgebra $A \subseteq C(X, E)$, equipped with some norm that makes it complete, such that (1) $A$ has as an element the constant function $x \mapsto 1_{E}$, (2) $A$ separates points on $X$, i.e. given distinct points $a, b \in X$, there exists $f \in A$ such that $f(a) \neq f(b)$ and (3) the evaluation map

$$
e_{x}:\left\{\begin{array}{l}
A \rightarrow E \\
f \mapsto f(x)
\end{array}\right.
$$

is continuous, for each $x \in X$. 
We remark that, as it stands, condition (3) is the very weak assumption that the inclusion map $A \hookrightarrow E^{X}$ is continuous, where $E^{X}$ is the given cartesian product topology, but it follows from the Closed Graph Theorem that if $A$ is an $E$-valued function algebra on $X$, then the inclusion map $A \hookrightarrow C(X, E)$ is continuous, so there exists some constant $M>0$ such that

$$
\|f\|_{X} \leq M\|f\|_{A}, \forall f \in A .
$$

Normally, we shall use the same notation $a$ for the element $a \in E$ and the constant function $x \mapsto a$ on $X$. The map $a \mapsto(x \mapsto a)$ imbeds $E$ isomorphically as a subalgebra of each $E$-valued function algebra $A$, and we normally identify $E$ with its image. Note that $A$ is commutative if and only if $E$ is commutative.

The classical concept of a function algebra (cf. [6, 4]) corresponds, in our terminology, to a $\mathbb{C}$-valued function algebra. Note, however, that some authors (e.g. [2]) have used the term function algebra to refer only to closed subalgebras of $C(X)$. We do not assume that an $E$-valued function algebra on $X$ is closed in the uniform norm.

An important class of examples is afforded by taking a compact set $X \subset \mathbb{C}^{n}$ and a commutative unital Banach algebra $E$, and defining the algebra $P(X, E)$ to be the uniform closure of $E[z] \mid X$ in $C(X, E)$, where $E[z]=E\left[z_{1}, \ldots, z_{n}\right]$ is the algebra of all polynomials in the coordinate functions $z_{1}, \ldots, z_{n}$ with coefficients in $E$. We can also form the algebra $R(X, E)$, defined to be the uniform closure on $X$ of the algebra of functions of the form $p(z) / q(z)$, where $p(z) \in E[z], q(z) \in E[z]$ and $q(x) \in E^{-1}$ whenever $x \in X$.

Johnson [9] considered the rather similar concept of the convolution algebra $L^{1}(G, A)$ of $A$-valued Bochner-integrable functions from a locally compact abelian group $G$ into a commutative Banach algebra $A$. The abstract Fourier transform maps such an $L^{1}(G, A)$ isomorphically to an $A$-valued algebra of continuous functions on the dual group $\hat{G}$.

There is also work [1] on operator-valued Fourier-Stieltjes algebras, and operatorvalued maps occur in applications such as homotopy theory, but in this paper we are going to concentrate on algebras of functions into commutative algebras $E$. Specifically, we shall study boundaries. We proceed to define the terms.

1.2. Characters. For a commutative unital Banach algebra $A$, let $M(A)$ denote the set of all characters (non-zero complex-valued multiplicative linear functionals) on $A$. It is well known that $M(A)$ is non-empty and that its elements are automatically continuous, with norm 1 . Endowed with the weak-star topology, $M(A)$ becomes a compact Hausdorff space. The Gelfand transform of $f \in A$ is the complex-valued function $\hat{f}$ defined by $\hat{f}(\varphi)=\varphi(f)$ on $M(A)$. Let $\hat{A}=\{\hat{f}: f \in A\}$. The algebra $\hat{A}$ consists of $\mathbb{C}$-valued continuous functions on $M(A)$. Hence, it is a $\mathbb{C}$-valued function algebra on $M(A)$ when endowed with the quotient norm. However, we shall use the notation $\|\hat{f}\|$ to denote the uniform norm of $\hat{f}$ on $M(A)$, and with respect to this norm $\hat{A}$ may or may not be complete.

The kernel of the map

$$
\text { ^ }\left\{\begin{array}{l}
A \rightarrow \hat{A} \\
f \mapsto \hat{f}
\end{array}\right.
$$


is the Jacobson radical of $A$. The characters on $C(X)$ are exactly the evaluations $e_{x}: f \mapsto f(x)$, with $x \in X$, and $X$ is homeomorphic to $M(C(X))$ with its relative weakstar topology as a subset of the dual $A^{*}$.

When $A$ is a $\mathbb{C}$-valued function algebra on $X$, the map $x \mapsto e_{x} \mid A$ imbeds $X$ homeomorphically as a compact subset of $M(A)$. When this map is surjective, one calls $A$ a natural $\mathbb{C}$-valued function algebra on $X$ [4].

The basic example $C(X, E)$ itself was studied by Hausner [7], who showed that its maximal ideal space is homeomorphic to $M(E) \times X$. More precisely, he showed [7, Lemma 2] the following.

LEMMA 1.1 [7]. For each commutative Banach algebra E with identity and each compact Hausdorff space $X$, the map $(\phi, x) \mapsto \phi \circ e_{x}$ is a homeomorphism from $M(E) \times$ $X$ onto $M(C(X, E)$.

\subsection{Shilov boundary and peak points.}

Definition 1.2. A closed boundary for a commutative Banach algebra $A$ is a closed subset $F \subseteq M(A)$ such that for each $a \in A$,

$$
\sup _{\varphi \in M(A)}|\hat{a}(\varphi)|=\sup _{\varphi \in F}|\hat{a}(\varphi)| .
$$

The Shilov boundary of $A$ is the intersection

$$
\Gamma(A)=\bigcap\{F: F \text { is a closed boundary for } A\} .
$$

It can be shown $([\mathbf{1 5}$, Theorem 15.2] or $[\mathbf{1 3}])$ that $\Gamma(A)$ is the unique minimal closed boundary for $A$.

DEFINITION 1.3. Let $A$ be a unital commutative Banach algebra. A closed subset $S \subseteq M(A)$ is called a peak set if there exists an element $a \in A$ such that $\hat{a}(\varphi)=1$ for $\varphi \in S$ and $|\hat{a}(\psi)|<1$ for $\psi \in M(A) \backslash S$. A point $\varphi \in M(A)$ is a peak point for $A$ if $\{\varphi\}$ is a peak set. We write $S_{0}(A)$ for the set of peak points for $A$.

Obviously, $S_{0}(A) \subseteq \Gamma(A)$. If $M(A)$ is metrisable, then (cf. [4, Cor. 4.3.7]) $\Gamma(A)$ is the closure of $S_{0}(A)$.

1.4. Main result. Our results are about commutative algebras.

In Section 2 we introduce the concept of an admissible quadruple $(X, E, B, \tilde{B})$, which formalises the idea of an $E$-valued function algebra $\tilde{B}$ that is organically connected to a $\mathbb{C}$-valued function algebra $B$ on the same space $X$. To such a quadruple we associate an injective map $\pi: M(E) \times X \rightarrow M(\tilde{B})$, and we say that the quadruple is natural when $\pi$ is bijective. We prove the following result about the relation between the three Shilov boundaries that are in play.

THEOREM 1.2. Let $(X, E, B, \tilde{B})$ be a natural admissible quadruple. Then the associated map $\pi$ maps $\Gamma(E) \times \Gamma(B)$ homeomorphically onto $\Gamma(\tilde{B})$

We give some specific examples, and other results. 


\section{Admissible Quadruples.}

Definition 2.1. By an admissible quadruple we mean a quadruple $(X, E, B, \tilde{B})$, where

(1) $X$ is a compact Hausdorff space,

(2) $E$ is a commutative Banach algebra with unit,

(3) $B \subseteq C(X)$ is a natural $\mathbb{C}$-valued function algebra on $X$,

(4) $\tilde{B} \subseteq C(X, E)$ is an $E$-valued function algebra on $X$,

(5) $B \cdot E \subseteq \tilde{B}$ and

(6) $\{\lambda \circ f, f \in \tilde{B}, \lambda \in M(E)\} \subseteq B$.

We remark that if we assume that the linear span of $B \cdot E$ is dense in $\tilde{B}$, then (6) is automatically true.

Condition (6) is undemanding if the Jacobson radical $J(E)$ of $E$ is large. In fact, we are mainly interested in semi-simple algebras. The meat of Theorem 1.2 is really about the quotient $E / J(E)$.

Given an admissible quadruple $(X, E, B, \tilde{B})$, we define the associated map

$$
\pi:\left\{\begin{array}{rl}
M(E) \times X & \rightarrow M(\tilde{B}) \\
(\psi, x) & \mapsto \psi \circ e_{x}
\end{array} .\right.
$$

LeMma 2.1. Let $(X, E, B, \tilde{B})$ be an admissible quadruple. Then the associated map $\pi$ is a continuous injection.

Proof. $\pi$ is injective from $M(E) \times X$ into $M(\tilde{B})$, since $\hat{E}$ separates points on $M(E)$ and $\hat{B}$ separates points on $X$. To see that $\pi$ is continuous, observe that it the composition of the (weak-star continuous) restriction map $C(X, E)^{*} \rightarrow(\tilde{B})^{*}$ with Hausner's homeomorphism $M(E) \times X \rightarrow M(C(X, E))$.

COROLlary 2.2. Let $(X, E, B, \tilde{B})$ be an admissible quadruple. Then the following are equivalent:

(1) The associated map $\pi$ is surjective.

(2) The associated map $\pi$ is bijective.

(3) The associated map $\pi$ is a homeomorphism of $M(E) \times X$ onto $M(\tilde{B})$.

Definition 2.2. We say that an admissible quadruple $(X, E, B, \tilde{B})$ is natural if the associated map $\pi$ is bijective.

For instance, if $B$ is a natural $\mathbb{C}$-valued function algebra on $X$, then $(X, \mathbb{C}, B, B)$ is a natural admissible quadruple, so this terminology is a reasonable extension of the usual use of 'natural'. Further, if $(X, E, B, \tilde{B})$ is an admissible quadruple and $E$ is semisimple, then $\hat{E}$ (with the induced norm given by $\|\hat{h}\|=\|h\|_{E}$ ) is a natural $\mathbb{C}$-valued function algebra on $M(E)$, so $\tilde{B}$ is isometrically isomorphic to a $\mathbb{C}$-valued function algebra on $M(E) \times X$, and it is a natural $\mathbb{C}$-valued function algebra if and only if the quadruple is natural.

Tomiyama [14] showed that if $A$ and $B$ are commutative Banach algebras with identity, and some completion of $C$ of $A \otimes B$ is also a Banach algebra, then the natural map $M(A) \times M(B) \rightarrow M(C)$ is a homeomorphism. Thus, if $(X, E, B, \tilde{B})$ is an admissible quadruple, and the linear span of $B \cdot E$ is dense in $\tilde{B}$, we may apply Tomiyama's theorem with $A=E$ and $B=B$ and deduce that the quadruple is natural. 
In view of the corollary, when given a natural admissible quadruple $(X, E, B, \tilde{B})$, we often identify $M(E) \times X$ with $M(\tilde{B})$.

Proof of Theorem 1.2. First, we show that the image of $\pi$ is a boundary for $\tilde{B}$ : Let $f \in \tilde{B}$. Fix a character $\phi \in M(\tilde{B})$. Then $\phi$ is of the form $\psi \circ e_{x}$ for some $x \in X$ and some $\psi \in M(E)$, and then $\hat{f}(\phi)=(\psi \circ f)(x)$. Now $\psi \circ f \in B$, so there exists a point $y \in \Gamma(B)$ such that $|(\psi \circ f)(x)| \leq|(\psi \circ f)(y)|$. Next, $(\psi \circ f)(y)=\widehat{f(y)}(\psi)$, and $f(y) \in E$, so there exists a point $\chi \in \Gamma(E)$ such that $|\widehat{f(y)}(\psi)| \leq|\widehat{f(y)}(\chi)|$. Thus,

$$
|\hat{f}(\phi)| \leq|\hat{f}(\pi(\chi, y))| .
$$

This shows that for each $f \in \tilde{B}, \hat{f}$ attains its maximum modulus on the image of $\pi$ so that image is a boundary, and

$$
\Gamma(\tilde{B}) \subseteq \pi(\Gamma(E) \times \Gamma(B)) .
$$

To see the opposite inclusion, fix $x \in \Gamma(B)$ and $\psi \in \Gamma(E)$. Let $U$ be any neighbourhood of $x$ in $X$ and $V$ be any neighbourhood of $\psi$ in $M(E)$. There exists $f \in B$ such that $\|\hat{f}\|=1$ and $|f(y)|<1$ for all $y \in X \backslash U$. In addition, there exists $v \in E$ such that $\|\hat{v}\|=1$ and $|\phi(v)|<1$ for all $\phi \in M(E) \backslash V$. Now define $g: X \rightarrow E$ by $g=v f$. We have $g \in \tilde{B}$ and

$$
\begin{aligned}
\|\hat{g}\| & =\sup _{\phi \in M(E)} \sup _{y \in X}\left|\widehat{v f}\left(\phi \circ e_{y}\right)\right| \\
& =\sup _{\phi \in M(E)} \sup _{y \in X}|f(y) \phi(v)| \\
& =\sup _{\phi \in M(E)}|\phi(v)| \cdot \sup _{y \in X}|f(y)| \\
& =\|\hat{v}\| \cdot\|\hat{f}\|=1 .
\end{aligned}
$$

On the other hand, every $\varphi^{\prime} \in \pi(M(E) \times X \backslash(U \times V))$ is of the form $\varphi^{\prime}=\phi \circ e_{y}$ with $y \in X \backslash U$ or $\phi \in M(E) \backslash V$ (or both). Therefore,

$$
\left|\hat{g}\left(\varphi^{\prime}\right)\right|=\left|\varphi^{\prime}(v f)\right|=|\phi(v) f(y)|<1 .
$$

Since $U$ and $V$ were arbitrary neighbourhoods, it follows from [15, Theorem 15.3] that $\psi \circ e_{x} \in \Gamma(\tilde{B})$. Therefore, $\pi(\Gamma(B) \times \Gamma(E)) \subseteq \Gamma(\tilde{B})$ and so the proof is complete.

2.1. Examples. (i) Let $X$ be a compact Hausdorff space and $E$ be a unital commutative Banach algebra. Then $(X, E, C(X), C(X, E))$ is an admissible quadruple. It is natural by Lemma 1.1, and this case of Theorem 1.2 is Hausner's theorem [7] that the Shilov boundary of $C(X, E)$ is equal to the cartesian product $X \times \Gamma(E)$.

(ii) Let $(X, d)$ be a compact metric space and $E$ be a commutative unital Banach algebra. For a constant $0<\alpha \leq 1$ and a function $f: X \rightarrow E$, the Lipschitz constant of $f$ is defined as

$$
p_{\alpha}(f):=\sup _{\substack{x, y \in X \\ x \neq y}} \frac{\|f(x)-f(y)\|}{d(x, y)^{\alpha}},
$$

and the $E$-valued big Lipschitz algebra or simply $E$-valued Lipschitz algebra (of order $\alpha)$ is defined by

$$
\operatorname{Lip}^{\alpha}(X, E)=\left\{f: X \rightarrow E: p_{\alpha}(f)<\infty\right\}
$$


Similarly, for $0<\alpha<1$, the $E$-valued little Lipschitz algebra (of order $\alpha$ ) is defined by

$$
\operatorname{lip}^{\alpha}(X, E)=\left\{f \in \operatorname{Lip}^{\alpha}(X, E): \frac{\|f(x)-f(y)\|}{d(x, y)^{\alpha}} \rightarrow 0 \text { as } d(x, y) \rightarrow 0\right\} .
$$

For each $f \in \operatorname{Lip}^{\alpha}(X, E)$ we define a norm by

$$
\|f\|_{\alpha}=\|f\|_{X}+p_{\alpha}(f) .
$$

It was shown in [3] that $\left(\operatorname{Lip}^{\alpha}(X, E),\|\cdot\|_{\alpha}\right)$ is a Banach algebra having $\operatorname{lip}^{\alpha}(X, E)$ as a closed subalgebra. It is relatively straightforward to check that $\left(X, E, \operatorname{Lip}^{\alpha}(X, \mathbb{C}), \operatorname{Lip}^{\alpha}(X, E)\right)$ is an admissible quadruple for each $\alpha \in(0,1]$, and that $\left(X, E, \operatorname{lip}^{\alpha}(X, \mathbb{C}), \operatorname{lip}^{\alpha}(X, E)\right)$ is an admissible quadruple for each $\alpha \in(0,1)$. (The result that the maximal ideal space of $\operatorname{Lip}^{\alpha}(X)$ is $X$ is originally due to Sherbert $[4,12]$.)

The scalar-valued Lipschitz algebras are normal because if $F$ and $K$ are disjoint non-empty closed subsets of $X$, then the function $f: x \mapsto \frac{d(x, F)}{d(x, F)+d(x, K)}$ belongs to $\operatorname{Lip}^{1}(X)$. It follows $[4$, p. 413] that they have partitions of unity subordinate to any open covering. Applying partitions of unity and a method similar to Hausner's in [7, Lemma 1], one can see that each of these $E$-valued Lipschitz algebras (and little Lipschitz algebras) is dense in $C(X, E)$. Then, given a character $\phi$ on $\operatorname{Lip}^{\alpha}(X, E)$ and a function $f \in C(X, E)$, we may choose a sequence $\left(f_{n}\right) \in \operatorname{Lip}^{\alpha}(X, E)$ such that $\left\|f-f_{n}\right\|_{X} \rightarrow 0$. Since

$$
\|\hat{h}\|_{M\left(\operatorname{Lip}^{\alpha}(X, E)\right)} \leq\|h\|_{X}
$$

for each $h \in \operatorname{Lip}^{\alpha}(X, E)$, the sequence $\left(\phi\left(f_{n}\right)\right)$ is Cauchy, so we may define $\tilde{\phi}(f)=$ $\lim _{n} \phi\left(f_{n}\right)$. Clearly, $\tilde{\phi}(f)$ does not depend on the choice of $\left(f_{n}\right)$, and $\tilde{\phi}$ is a well-defined character on $C(X, E)$, extending $\phi$. Thus, by Lemma $1.1, \phi=\psi \circ e_{x}$ for some $\psi \in$ $M(E)$ and some $x \in X$. A similar argument works for $\operatorname{lip}^{\alpha}(X, E)$. Thus, Theorem 1.2 applies, and the Shilov boundary of $\operatorname{Lip}^{\alpha}(X, E)\left(\right.$ or $\left.\operatorname{lip}^{\alpha}(X, E)\right)$ is equal to the cartesian product $X \times \Gamma(E)$ in the product topology.

(iii) Let $X$ be a compact set in $\mathbb{C}^{n}$ and $E$ be a unital commutative Banach algebra, and consider the algebra $P(X, E)$. The algebra $P(X)=P(X, \mathbb{C})$ has character space naturally identified with $\hat{X}$, the polynomially convex hull of $X[\mathbf{2}, \mathbf{6}, \mathbf{1 1}, \mathbf{1 3}]$, and $P(X, E)$ may be regarded as an $E$-valued function algebra on $\hat{X}$. Using this, it is easy to see that $(\hat{X}, E, P(X), P(X, E))$ is an admissible quadruple: In fact, each $f \in P(X, E)$ is the limit in norm of a sequence $\left\{g_{n}\right\}$ with each $g_{n}$ of the form $\sum_{j=1}^{m_{n}} a_{j} p_{j}$, where $m_{n} \in n, a_{j} \in E$ and $p_{j} \in P(X)$ depend on $n$. Then by a method similar to [10, Proposition 1.5.6] one sees that $P(X, E)=P(X) \check{\otimes} E$. Thus, since $P(X)=P(\hat{X})$ [6, Chapter II, Theorem 1.4], we have

$$
P(X, E)=P(X) \check{\otimes} E=P(\hat{X}) \check{\otimes} E=P(\hat{X}, E) .
$$

We note that in the particular case when $X$ is a compact plane set, $\hat{X}$ is obtained by 'filling in the holes' in $X$, and $\Gamma(P(X))$ is the topological boundary of $\hat{X}$ in $\mathbb{C}$. In higher dimensions, the Shilov boundary $\Gamma(P(X))$ is some closed subset of bdy $(X)$.

By a method similar to [7, Lemma 2], one sees that every character $\phi$ on $P(X, E)$ is of the form $\phi=\psi \circ e_{x}$ for some $x \in \widehat{X}$ and some $\psi \in M(E)$. Therefore the theorem applies, and the Shilov boundary of $P(X, E)$ is equal to the cartesian product $\Gamma(E) \times$ $\Gamma(P(X))$. 
(iv) Let $X \subset \mathbb{C}$ be compact, $E$ be a commutative unital Banach algebra and $E^{*}$ be the dual space of $E$. The algebra of $E$-valued analytical functions is defined as follows:

$$
A(X, E)=\left\{f \in C(X, E): \Lambda \circ f \in A(X), \Lambda \in E^{*}\right\},
$$

where $A(X)$ is the algebra of all continuous functions on $X$ into $\mathbb{C}$ which are holomorphic on the interior of $X$. It is clear that $A(X, E)$ is a closed subalgebra of $\left(C(X, E),\|\cdot\|_{X}\right)$. Arens showed [6] that $M(A(X))$ is naturally identified with $X$, and so one sees at once that $(X, E, A(X), A(X, E))$ is an admissible quadruple. By a method similar to the one given in [5, Theorem 2], we can deduce that when $E$ is a unital Banach algebra then every character $\phi$ on $A(X, E)$ is of the form $\phi=\psi \circ e_{x}$ for some $x \in X$ and some $\psi \in M(E)$. So the Shilov boundary of $A(X, E)$ is equal to the cartesian product $\Gamma(A(X)) \times \Gamma(E)$ in the product topology by Theorem 1.2.

(v) Theorem 1.2 also applies to the algebra $R(X, E)$ for any commutative unital Banach algebra $E$. The characters on $R(X)$ are the evaluations at the points of the rationally convex hull $\check{X}$ of $X$, which is the set of points $a \in \mathbb{C}^{n}$ such that each polynomial $p(z) \in \mathbb{C}[z]$ that vanishes at $a$ also vanishes at some point of $X$. In dimension $n=1, \check{X}=X$, but in higher dimensions it may be a larger set. So $R(X)$ is a natural $\mathbb{C}$-valued function algebra on $\check{X}$.

We claim that every character $\phi$ on $R(X, E)$ is of the form $\phi=\psi \circ e_{x}$, for some $\psi \in M(E)$ and some $x \in \check{X}$.

To see this, let $\phi \in M(R(X, E))$. The restriction of $\phi$ to $P(X, E)$ is a character, so there exists $x_{0} \in \hat{X}$ and $\psi \in M(E)$ such that $\phi=\psi \circ e_{x_{0}}$ on $P(X, E)$. Given $g=p / q$ where $p, q \in E[z]$ and $q(x) \in E^{-1}$ for each $x \in X$, we get $p=g q, \phi(p)=\phi(g) \phi(q)$, and hence $\left(\right.$ since $\left.\phi(q)=\psi\left(q\left(x_{0}\right)\right) \neq 0\right)$

$$
\phi(g)=\frac{\psi\left(p\left(x_{0}\right)\right)}{\psi\left(q\left(x_{0}\right)\right)}=\psi\left(g\left(x_{0}\right)\right) .
$$

Thus, by continuity, $\phi=\psi \circ e_{x_{0}}$ on all $R(X, E)$. Since $R(X) 1 \subseteq R(X, E)$, it follows that $x_{0} \in \check{X}$ (cf. [6, Theorem 5, p. 86]. Thus, the claim holds.

Hence, if $X$ is rationally convex, then $(X, E, R(X), R(X, E))$ is a natural admissible quadruple.

There seems no reason to suppose that $R(X, E)=R(\check{X}, E)$ for general $X$, except when $E$ is a uniform algebra. In general, one readily sees that there is a contractive algebra homomorphism

$$
R(X, E) \rightarrow C(\check{X}, C(M(E)))
$$

and that if $E$ is a uniform algebra then this gives an isometric isomorphism from $R(X, E)$ onto $R(\check{X}, E)$. We do not, however, know an example in which the restriction $\operatorname{map} R(\check{X}, E) \rightarrow R(X, E)$ is not onto.

(vi) The bidisk algebra [2] may (in view of Hartogs' theorem) be regarded as $\tilde{B}=A(X, A(X))$, where $X$ is the closed unit disk in $\mathbb{C}$. The quadruple $(X, A(X), A(X), \tilde{B})$ is admissible, the theorem applies, and reduces to the classical fact that the Shilov boundary of $\tilde{B}$ is the torus. More generally, one gets the (known) result that the Shilov boundary of $A(X, A(Y))$ is bdy $X \times$ bdy $Y$ whenever $X \subset \mathbb{C}$ and $Y \subset \mathbb{C}$ are compact.

(vii) Let $0<\alpha<1$. The subalgebra of $\operatorname{lip}^{\alpha}(X, E)$, which is the closure of $E[z] \mid X$ in $\operatorname{Lip}^{\alpha}(X, E)$ norm, where $X \subset \mathbb{C}^{n}$, is denoted by $\operatorname{Lip}_{P}^{\alpha}(X, E)$. It is easy to see that $\operatorname{Lip}_{P}^{\alpha}(X, E)$ is dense in $P(X, E)$. Now by [8, p. 15], $M\left(\operatorname{Lip}_{P}^{\alpha}(X, \mathbb{C})\right)=\hat{X}$. Thus, if $X$ is 
polynomially convex, then the quadruple $\left(X, E, \operatorname{Lip}_{P}^{\alpha}(X, \mathbb{C}), \operatorname{Lip}_{P}^{\alpha}(X, E)\right)$ is admissible and natural.

(viii) Also, for $0<\alpha<1$, the subalgebra of $\operatorname{lip}^{\alpha}(X, E)$, which is the closure of the algebra of functions of the form $p(z) / q(z)$ in $\operatorname{Lip}^{\alpha}(X, E)$, where $X \subset \mathbb{C}^{n}, p(z) \in E[z]$, $q(z) \in E[z]$, and $q(x) \in E^{-1}$ whenever $x \in X$, is denoted by $\operatorname{Lip}_{R}^{\alpha}(X, E)$. It is easy to see that $\operatorname{Lip}_{R}^{\alpha}(X, E)$ is dense in $R(X, E)$. Now by [8, p. 15], $M\left(\operatorname{Lip}_{R}^{\alpha}(X, \mathbb{C})\right)=\check{X}$. Thus, if $X$ is rationally convex, then the quadruple $\left(X, E, \operatorname{Lip}_{R}^{\alpha}(X, \mathbb{C}), \operatorname{Lip}_{R}^{\alpha}(X, E)\right)$ is admissible and natural.

2.2. Peak points. By similar arguments, one obtains the following.

THEOREM 2.3. Let $(X, E, B, \tilde{B})$ be a natural admissible quadruple. Then the set of peak points of $\tilde{B}$ is equal to the cartesian product $S_{0}(B) \times S_{0}(E)$ in the product topology, that is,

$$
S_{0}(\tilde{B})=S_{0}(B) \times S_{0}(E)
$$

ACKNOWLEDGEMENTS. This paper was conceived while the first author was visiting National University of Ireland, Maynooth, whose hospitality is acknowledged with thanks. The authors are grateful to the referee, whose observations and suggestions greatly improved the paper.

\section{REFERENCES}

1. G. A. Bagheri-Bardi, A. R. Medghalchi and N. Spronk, Operator-valued convolution algebras, Houston J. Math. 36 (4) (2010), 1023-1036.

2. A. Browder, Function algebras (Benjamin, New York, NY, 1969).

3. H. X. Cao, J. H. Zhang and Z. B. Xu, Characterizations and extensions of Lipschitz- $\alpha$ operators, Acta Math. Sin. Engl. Ser. 22 (3) (2006), 671-678.

4. H. G. Dales, Banach algebras and automatic continuity, LMS Monographs, 24 (Clarendon Press, Oxford, UK, 2000).

5. S. Dierolf, K.-H. Schröder and J. Wengenroth, Characters on certain function algebras, Funct. Approx. Comment. Math. 26 (1998) 53-58.

6. T. W. Gamelin, Uniform algebras (Prentice-Hall, Englewood Cliffs, NJ, 1969). $246-249$

7. A. Hausner, Ideals in a certain Banach algebra, Proc. Amer. Math. Soc. 8 (2) (1957),

8. T. G. Honary and H. Mahyar, Approximation in Lipschitz algebras, Quaest. Math. 23 (1) (2000), 13-19.

9. G. P. Johnson, Spaces of functions with values in a Banach algebra, Trans. Amer. Math. Soc. 92 (1959) 411-429.

10. E. Kaniuth, A course in commutative Banach algebras, Graduate Texts in Mathematics, 246 (Springer, New York, NY, 2009). 1970).

11. G. Leibowitz, Lectures on complex function algebras (Scott Foresman, Glenview, IL,

12. D. R. Sherbert The structure of ideals and point derivations in Banach algebras of Lipschitz functions, Trans. AMS 111 (1964), 240-272.

13. E. L. Stout, The theory of uniform algebras (Bogden and Quigley, Tarrytown-onHudson, NY, 1971).

14. J. Tomiyama, Tensor products of commutative Banach algebras, Tohoku Math. J. 12 (1960) $147-154$.

15. W. Zelazko, Banach algebras (Elsevier, New York, NY, 1973). 